\title{
UM SÉCulo de Medições de PH
}

\section{Filomena Camões*}

\begin{abstract}
"...exemplo perfeito para muitos que, nesta era das velocidades, afirmando-se cientistas, servem mal a Ciência, trocando-a pelo superficial e espectacular"
\end{abstract}

E. K. Rideal

in Obituário de Søren Peter Lauritz Sørensen, 1939

A primeira década do século $X X$ foi palco de diversos e importantes avanços científicos em Química, despoletados, em particular, em 1884, pela Teoria da Dissociação Electrolítica - Tese de Doutoramento de S. Arrhenius (1859-1927), Prémio Nobel da Química de 1903. Alguns deles estão particularmente associados ao reconhecimento da relevância dos ácidos, como substâncias fornecedoras de iões hidrogénio, no controlo do comportamento de reacções químicas, a que está ligado o nome de Søren
Peter Lauritz Sørensen (1868-1939), Assistente do Laboratório de Química do Instituto Politécnico da Dinamarca, de 1892 a 1901, e Director do Departamento de Química da empresa cervejeira Carlsberg, de 1901 a 1938.

Sørensen conduziu investigação verdadeiramente pioneira em quatro áreas: Síntese de aminoácidos, Procedimentos analíticos, Concentração hidrogeniónica e Propriedades das proteínas, em consequência do que publicou "Estudos de Enzimas I - Tra- tado sobre a Titulação de Formol" com quatro partes.

A primeira parte trata do método electrométrico para determinação da concentração hidrogeniónica e apresenta uma descrição exaustiva impressionante das fontes de erro. Igualmente rigorosa e exemplar é a determinação da constante de dissociação da água. A segunda abarca investigação sobre a preparação de soluções tampão padrão, de utilização generalizada, Figura 1.

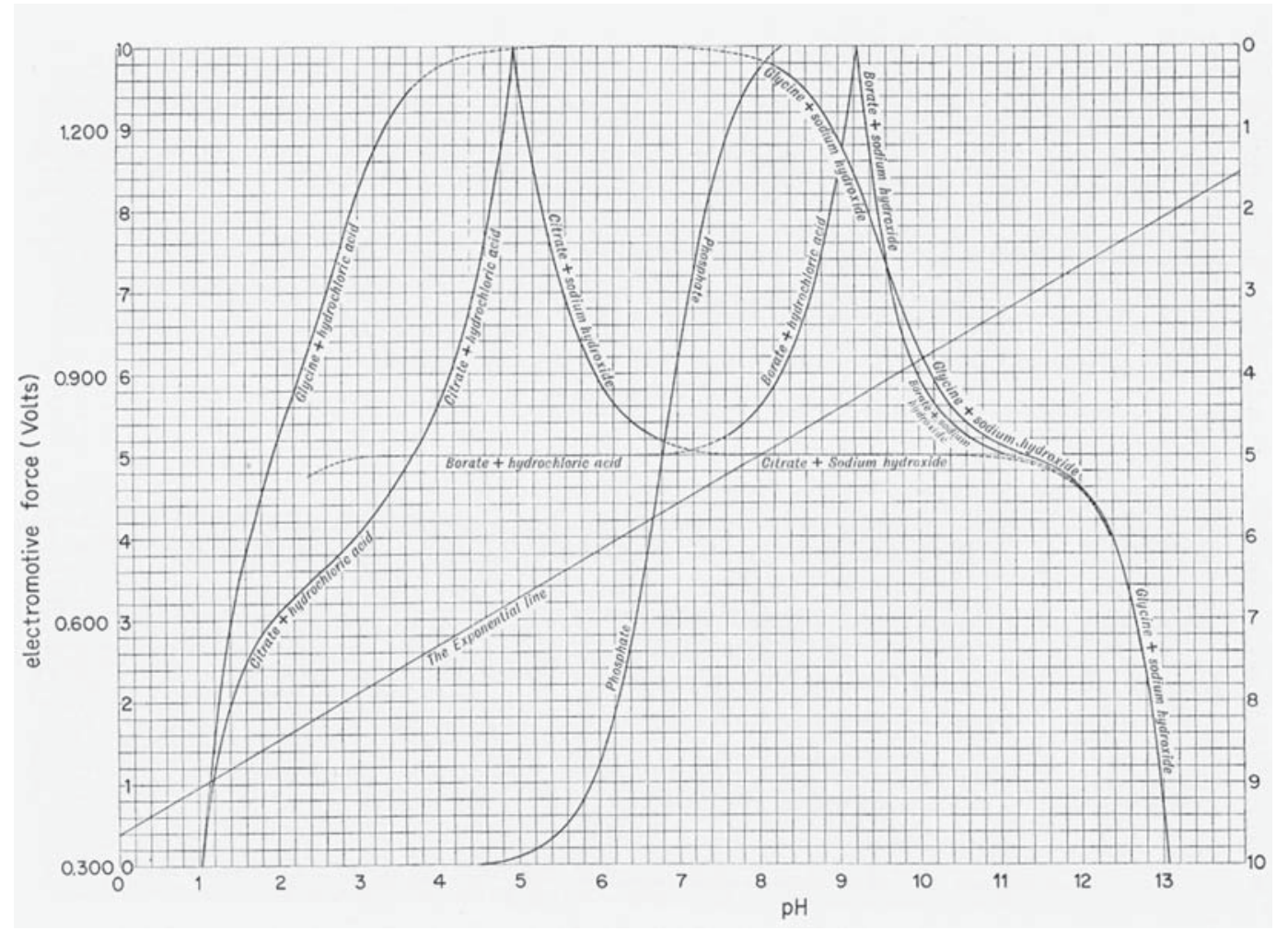

Figura 1 - Carta dos tampões de Sørensen que durante muitos anos foi utilizada como referência de pH. O sistema consiste numa série de pares de soluções, que misturadas nas quantidades indicadas nos eixos de ordenadas da esquerda e da direita atingem o valor de $\mathrm{pH}$ indicado em abcissa. (The Carlsberg Laboratory -1876/1976, Ed.H. Holter and K. Max Møller, RHODOS Publishing House, Copenhagen, 1976) 
A terceira trata do método colorimétrico para medição de acidez, das mais variadas soluções, Figura 2. A quarta trata da aplicação destes métodos ao estudo da catálise enzimática, Figura 3 , de interesse particular na optimização da acção da levedura da cerveja.
Por questões práticas de simplificação de escrita e manipulação de valores de concentração hidrogeniónica, $c_{H}$, capazes de abranger várias ordens de grandeza, mas de forma geral baixas, expressas por potências de dez de expoente negativo, $c_{H}=10^{-x}$,

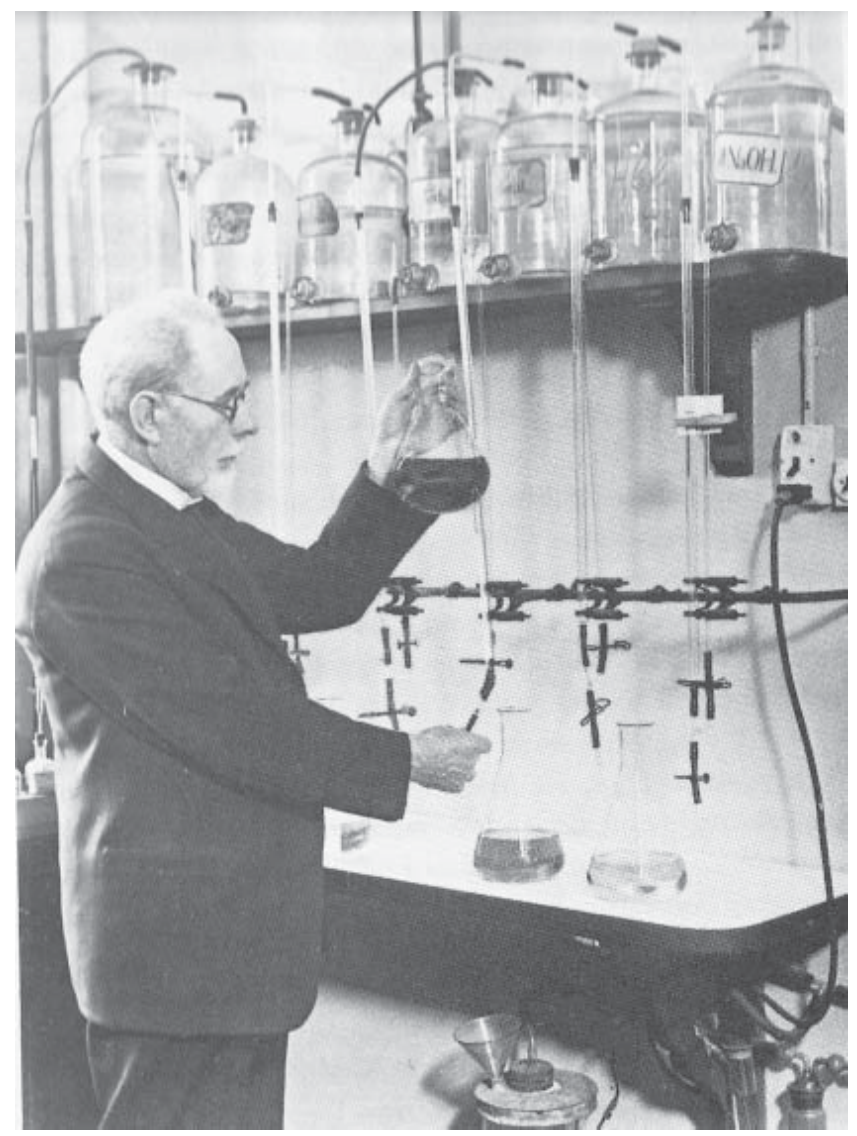

Figura 2 - Sørensen (1936) a executar titulações com o conjunto de soluções tampão e indicadores que desenvolveu. Debaixo da bancada de tampo em opala, é visível a parte superior do gerador electrolítico de hidrogénio gasoso. (The Carlsberg Laboratory-1876/1976, Ed.H. Holter and K. Max Møller, RHODOS Publishing House, Copenhagen, 1976)
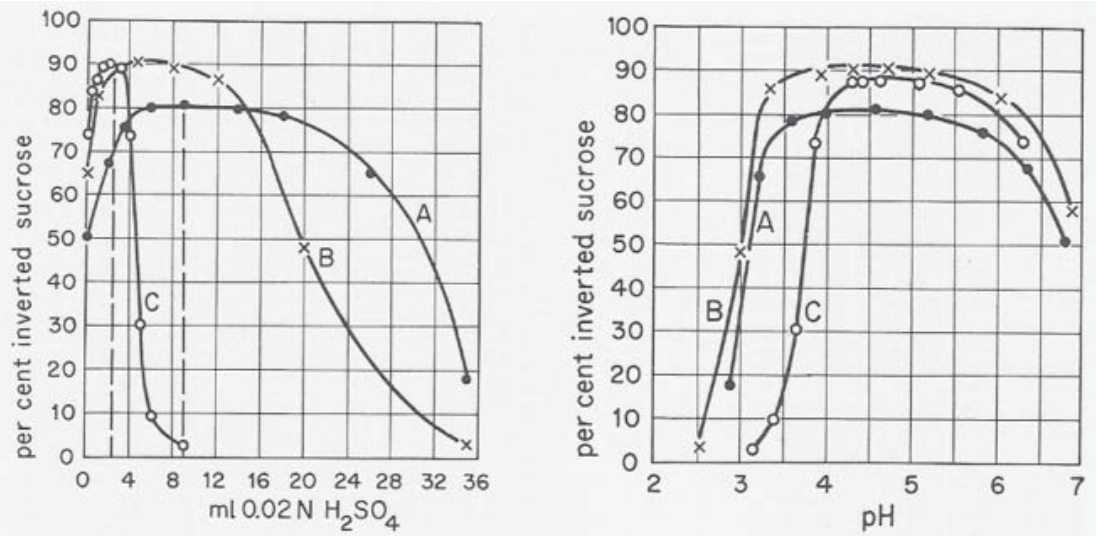

Figura 3 - Efeito da adição de ácido na actividade da invertase. A curva da esquerda indica a quantidade de ácido adicionado.

A curva da direita está representada de acordo com a nova escala logarítmica, $\mathrm{pH}$, de Sørensen. (The Carlsberg Laboratory-1876/1976, Ed.H. Holter and K. Max Møller, RHODOS Publishing House, Copenhagen, 1976)

Sørensen propôs, em 1909, que se usasse como uma medida da acidez do meio aquoso, o expoente multiplicado por $-1, \mathrm{x}$, o que é algebricamente equivalente ao logaritmo negativo do valor da concentração hidrogeniónica, - $\log c_{H}=x$, e a que chamou $\mathrm{pH}$; passava assim a lidar com números pequenos e geralmente positivos. A utilização da letra $\mathbf{p}$ resultava assim de a nova grandeza ser um expoente ou potência de base dez do valor da concentração hidrogeniónica, "pondus Hydrogenii", sendo curioso verificar que $\boldsymbol{p}$ é também a primeira letra das palavras equivalentes em várias outras línguas, ex: power, potenz, potence, etc. A utilização do operador algébrico, $p=-$ log, generalizou-se para outras espécies e grandezas químicas. Com a introdução do conceito de actividade, que reflecte o afastamento das espécies a comportamentos ideais, e com a publicação da Teoria de Interacção lónica, de Debye e Hückel, Sørensen e K. LinderstrømLang sugeriram, em 1910, uma nova definição de $\mathrm{pH}$ em termos da actividade hidrogeniónica, $a_{H}$ em solução aquosa, $\mathrm{pH}=-\log a_{\mathrm{H}}$.

É interessante verificar que o primeiro trabalho de revisão, 1914, sobre pH, é um livro da autoria da médica alemã Leonor Michaelis, que realça a importância dos iões hidrogénio e da sua medição em Biologia, o que é compreensível, sabendo como se sabe a dependência de $\mathrm{pH}$ dos processos fisiológicos. Não sendo fácil demonstrar a forma como o conhecimento da relevância de $\mathrm{pH}$ nos processos biológicos terá actuado como estímulo na investigação moderna em Bioquímica, afirma-se, no entanto, que transformou a Bioquímica numa ciência exacta. A investigação criteriosa de Sørensen levou-o a conseguir, em 1917, a cristalização da albumina do ovo, um passo determinante na caracterização das proteínas. Sørensen notou alterações de cor de determinados indicadores ácido-base corados, produzidas na presença de proteínas, sugerindo um desvio de $\mathrm{pH}$, geralmente para valores mais alcalinos do que realmente são. Este "erro das proteínas", se por um lado dificulta a medição de $\mathrm{pH}$ de fluidos fisiológicos com indicadores corados, por outro proporciona um método de detecção de proteínas, 
com tiras de papel impregnado com indicador tamponado. Com pH constante, o indicador apresenta uma cor na ausência de proteínas e outra na sua presença.

Mas não bastava ter uma definição conceptual de $\mathrm{pH}$; era necessário ter um método experimental recomendado para avaliação da grandeza, já que Sørensen, tal como outros, verificou que a detecção colorimétrica não apresenta sensibilidade suficiente para variações de $\mathrm{pH}$ inferiores a 0.5 , o que a torna inadequada para fins quantitativos.

Ao mesmo tempo que apresentou o conceito de $\mathrm{pH}$, Sørensen propôs uma metodologia para a sua medição, baseada num procedimento electrométrico anteriormente desenvolvido por Bjerrum e na lei analítica de Nernst, inicialmente empírica e posteriormente suportada teoricamente pela Termodinâmica de Gibbs.

Sørensen calculou $\mathrm{pH}$ a partir do potencial de uma célula electroquímica (inventadas por A. Volta em 1800) a funcionar em modo potenciométrico (situação de reversibilidade, i.e. corrente nula), constituída por um eléctrodo de calomelanos $\left(\mathrm{Hg}, \mathrm{Hg}_{2} \mathrm{Cl}_{2}\right)$, introduzido por Ostwald em 1894, sensível a $\mathrm{Cl}^{-}$, em $0.1 \mathrm{~mol} \mathrm{dm}^{-3} \mathrm{KCl}$ e um eléctrodo de hidrogénio gasoso $\left(\mathrm{Pt}, \mathrm{H}_{2}\right)$, sensível a $\mathrm{H}^{+}$, desenvolvido por Le Blanc, à pressão de hidrogénio de $1 \mathrm{~atm}$ e à temperatura de $18{ }^{\circ} \mathrm{C}$, mergulhado na solução cujo $\mathrm{pH}$ se pretendia medir:

$\mathrm{Pt}, \mathrm{H}_{2} \mid$ Sol. $\left(\mathrm{c}_{\mathrm{H}}\right)_{\mathrm{x}}$ ॥ Salt Bridgell $0.1 \mathrm{~mol}$ $\mathrm{dm}^{-3} \mathrm{KCl} \mid \mathrm{Hg}_{2} \mathrm{Cl}_{2}, \mathrm{Hg}$

O contacto entre as duas semi-células é efectuado através de uma ponte salina, "IPonte salina II.

A equação de Nernst, ainda empírica à altura, foi usada como lei analítica para relacionar o potencial medido, $E_{i}$, com o pH da solução onde o eléctrodo gasoso de hidrogénio mergulhava, $\left(c_{\mathrm{H}}\right)_{i}$ :

$$
E_{1}-E_{2}=\frac{R T}{F} \ln \frac{\left(C_{H}\right)_{2}}{\left(C_{H}\right)_{1}}=\frac{R T}{F} \ln 10 \lg \frac{\left(C_{H}\right)_{2}}{\left(C_{H}\right)_{1}}
$$

A letra $R$ é usada para a constante dos gases perfeitos, $F$ é a constante de Faraday e $T$ é a temperatura abso- luta. $\frac{R T}{F} \ln 10$, variação do potencial quando a concentração de ião hidrogénio varia de uma ordem de grandeza, é conhecida como o declive de Nernst, igual a $59.16 \mathrm{mV}$ a $25^{\circ} \mathrm{C}$.

Reconhecendo que nem a equação empírica de Nernst expressa em concentrações, nem a sua correspondente versão termodinâmica exprimindo actividades, levavam em conta a contribuição do potencial residual de junção líquida (diferença de potencial de junção líquida estabelecida em cada uma das duas junções entre o electrólito da ponte e o electrólito de cada semi-célula), Sørensen admitiu que o valor obtido não seria exactamente $\mathrm{pH}=-\log c_{\mathrm{H}}$ nem $\mathrm{pH}=-\log a_{\mathrm{H}}$, mas uma quantidade convencional $\mathrm{psH}$, que encontrou grande aceitação pela comunidade científica.

Para $1 \mathrm{~mol} \mathrm{dm}^{-3} \mathrm{HCl}$, Sørensen reportou o valor de potencial de célula $0.338 \mathrm{~V}$, em relação ao qual foram atribuídos valores de $\mathrm{pH}$ a inúmeras soluções:

$$
p s H=\frac{E-0.338}{0.05916}
$$

As medições de $\mathrm{pH}$ com o eléctrodo gasoso de hidrogénio em base de Pt (ou Pd) são as de mais elevada qualidade metrológica, usadas para atribuição de valores de referência a soluções tampão de $\mathrm{pH}$ primários ou secundários (Figura 4).

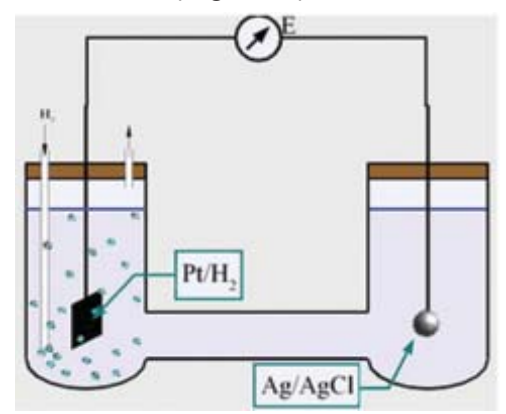

Figura 4 - Representação esquemática de circuito potenciométrico com célula de Harned constituída por eléctrodo gasoso de hidrogénio e eléctrodo de prata/cloreto de prata (adaptado de P. Spitzer, PTB-De)

R. Bates conduziu abundantes estudos sobre soluções tampão de pH por um processo convencional baseado na chamada célula de Harned, sem junção líquida:

Pt $\mathrm{H}_{2}(1 \mathrm{~atm})$ |solução tampão de $\mathrm{pH} ; \mathrm{KCl}(m)|\mathrm{AgCl}| \mathrm{Ag}$ $\left(m_{\mathrm{KCl}}=0.005 ; 0.01 ; 0.015 \mathrm{~mol} \mathrm{~kg}^{-1}\right)$ a que corresponde a reacção de célula

$$
1 / 2 \mathrm{H}_{2}+\mathrm{AgCl} \rightarrow \mathrm{Ag}+\mathrm{H}^{+}+\mathrm{Cl}^{-}
$$

O potencial de célula, $E$, permite calcular a função de acidez $\mathrm{p}\left(a_{\mathrm{H}} a_{\mathrm{Cl}}\right)$

$\mathrm{p}\left(a_{\mathrm{H}} a_{\mathrm{Cl}}\right)=-\lg \left(a_{\mathrm{H}} a_{\mathrm{Cl}}\right)=\left(E-E^{\circ}\right) /[(R T / F)$ In 10] $+\lg \left(m_{\mathrm{Cl}} / m^{\circ}\right)$

que, por um processo convencional, que recorre ao modelo de DebyeHückel, com a convenção de BatesGuggenheim, de soluções electrolíticas de força iónica inferior a $0.1 \mathrm{~mol}$ $\mathrm{kg}^{-1}$, conduz ao $\mathrm{pH}$ da solução tampão em estudo, $\mathrm{pH}=\mathrm{paH}$.

As letras têm o significado usual, sendo $m$ para concentração e a para actividade. $E^{\circ}$ é o potencial correspondente a uma célula contendo $\mathrm{HCl}$ em condições padrão, de actividade unitária.

A utilização do eléctrodo gasoso de hidrogénio, em rotina, além de pouco prática, pode ser mesmo inviável pela presença de interferentes.

A investigação sobre materiais sensíveis a hidrogenião levou a eleger o vidro Cremer, 1906, como alternativa prática aconselhada na grande maioria das situações, o que deu origem à ampla utilização de eléctrodos de vidro indicadores de $\mathrm{pH}$. Durante muitos anos, o vidro Corning 015, com a composição $\mathrm{Na}_{2} \mathrm{O}$ (21.4 mol\%), $\mathrm{CaO}$ $(6.4 \%)$, e $\mathrm{SiO}_{2}(72.2 \%)$, foi considerado o melhor eléctrodo de vidro com resposta idêntica ao eléctrodo gasoso de hidrogénio para soluções de $\mathrm{pH}$ entre 1 e 9, conforme a equação de Nernst. Apesar de investimento no estudo de outros vidros que pudessem observar a resposta teórica em todas as soluções e em toda a gama de valores práticos de $\mathrm{pH}$ tal não foi atingido. Existem desvios na zonas ácida e alcalina, estes explicados por selectividade do vidro não só aos iões hidrogénio, mas também aos iões alcalinos e alcalino-terrosos.

Um eléctrodo de vidro é geralmente constituído por uma membrana de cerca de $0.2 \mathrm{~mm}$ de espessura, geralmente esférica, soprada de vidro de sílica com óxidos metálicos que lhe 
conferem uma estrutura menos compacta, mais condutora, selada num tubo de vidro isolador. Mergulhando a esfera em água há novas quebras de ligação na estrutura do vidro e os espaços são preenchidos com água e os seus iões; há um aumento visível de dimensão da esfera, que corresponde a ter-se formado uma camada gelatinosa onde existe um potencial de iões hidrogénio, que confere ao eléctrodo a capacidade de estabelecer diferenças de potencial com iões hidrogénio existentes nas soluções aquosas em que mergulhe. A esfera de vidro tem duas superfícies, uma externa, e uma interna em contacto com uma solução aquosa de $\mathrm{pH}$ constante, $0.1 \mathrm{~mol} \mathrm{dm}^{-3}$ $\mathrm{HCl}$, em que mergulha um eléctrodo de referência interno de prata/cloreto de prata, destinado a um potencial constante e a permitir estabelecer ligação eléctricas com o circuito eléctrico de medição. $O$ eléctrodo de vidro simples é, afinal uma montagem de eléctrodos:

$\mathrm{Ag}|\mathrm{AgCl}| 0.1 \mathrm{~mol} \mathrm{dm}^{-3} \mathrm{HCl} \mid$ Vidro

Representação convencional do Eléctrodo de vidro simples

A medição de qualquer potencial é sempre efectuada relativamente a um eléctrodo de referência externa, geralmente outro eléctrodo de prata/cloreto de prata. As medições práticas de $\mathrm{pH}$, resultam de medidas de potencial de células onde há normalmente uma junção líquida, "I, entre os electrólitos das semi-células de cada um dos dois eléctrodos,

Eléctrodo de vidro|solução cujo $\mathrm{pH}$ se pretende medir $\| \mathrm{KCl} \geq 3.5 \mathrm{~mol} \mathrm{dm}^{-3}$ | Ag,AgCl

Conjuntos com os dois eléctrodos acoplados numa mesma montagem, designados por eléctrodos de vidro combinados, existem no comércio das várias marcas e são de ampla utilização, Figura 5.

Atendendo à elevada resistência dos eléctrodos de vidro, da ordem das dezenas ou centenas de $M \Omega$, a avaliação quantitativa de $\mathrm{pH}$ carece de circuitos de medição com amplificação de corrente, o que constituiu um atraso na utilização da técnica potenciométrica. Um aparelho medidor de
pH desenvolvido por Arnold Beckman (1935) contribuiu para divulgar um método de medição que é ao mesmo tempo de simples execução em rotina e de qualidade adequada para as áreas de investigação científica em que são feitos estudos em função do $\mathrm{pH}$ do meio.

Medições com aparelhos medidores de $\mathrm{pH}$ requerem calibração experimental. A calibração é feita fazendo corresponder os valores medidos de potencial da célula e o $\mathrm{pH}$ conhecido das soluções tampão de referência comerciais certificadas, mais ou menos adequadas ao problema analítico em estudo. Na generalidade das situações, a calibração é efectuada com dois ou mais dos tampões vulgarmente conhecidos como "tampões NIST", por alusão a uma das mais prestigiadas instituições que os produz e comercializa. Em diferentes sectores de actividade, por exemplo análises clínicas, são usadas soluções tampão de referência convencionalmente seleccionadas.

Matrizes complexas de elevada força iónica e/ou contendo proteínas, são

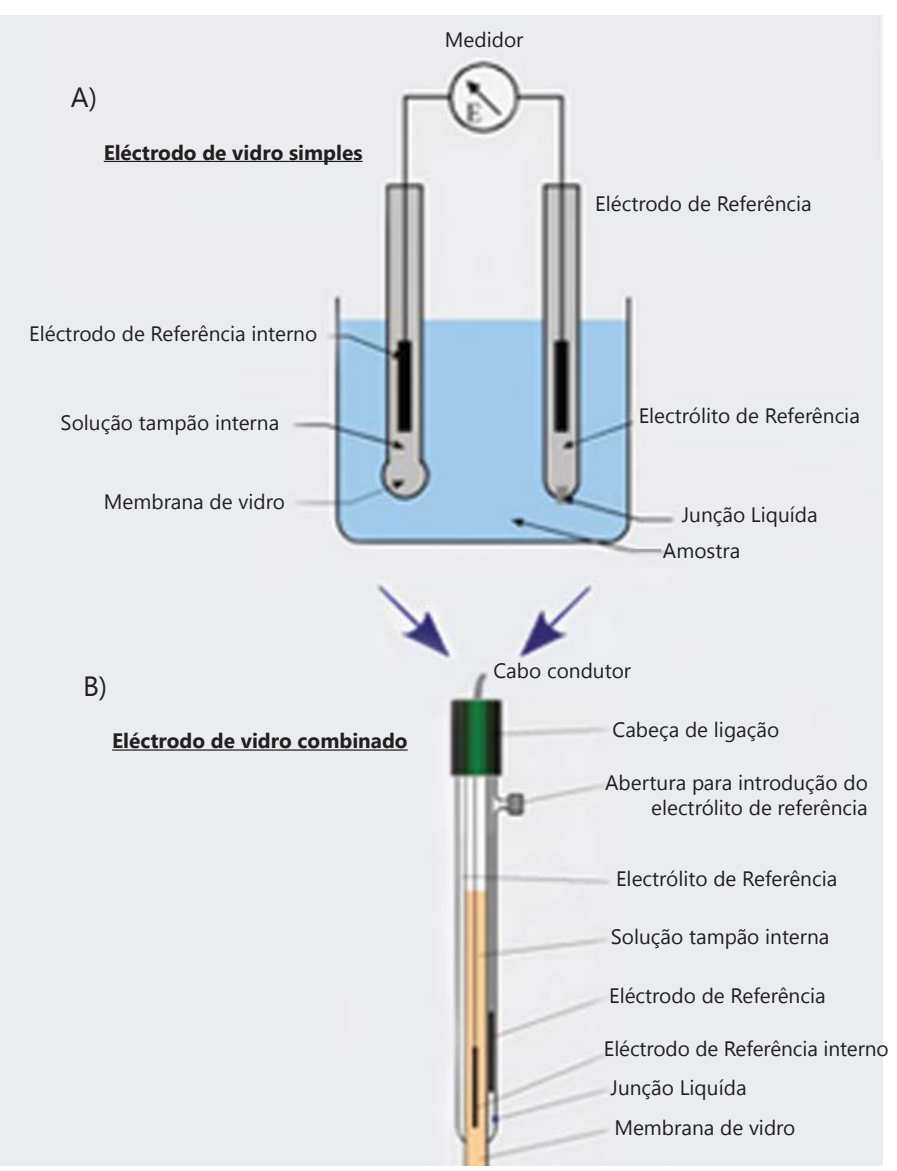

Figura 5 - Esquema do Eléctrodo de vidro, A) simples, com eléctrodo de referência externo; B) combinado, com eléctrodo de referência acoplado (adaptado de P. Spitzer, PTB-De) exemplos de situações em que a investigação científica procura ainda respostas plenamente satisfatórias.

No entanto, o facto de existirem aparelhos medidores de $\mathrm{pH}$ de preço acessível, sensíveis, robustos e cuja utilização não exige grande formação técnica, teve como consequência a generalização, ou mesmo banalização, da medição de $\mathrm{pH}$, não acompanhada da compreeensão das limitações da aplicabilidade do método e da compreensão do significado correcto da grandeza.

Sendo $\mathrm{pH}$ o parâmetro químico mais medido, é também, e até por isso, um tema em que é muitas vezes enganadora a ideia de que tudo é simples e sabido. Por ocasião da celebração do primeiro centenário da introdução do conceito de $\mathrm{pH}$ por Sørensen, continua a ser pertinente a citação de G. Mattock, 1963,

"...pH measurement is often deceptively easy ...

$\mathrm{pH}$ measurement can also be exasperatingly difficult." 